\title{
Selective autophagy: Lysophagy
}

Junya Hasegawa, ${ }^{\mathrm{a}, \mathrm{b}}$, Ikuko Maejima ${ }^{\mathrm{b}, 1}$, Ryo Iwamoto ${ }^{\mathrm{a}}$ and Tamotsu Yoshimori ${ }^{\mathrm{a}, \mathrm{b}}$

${ }^{a}$ Laboratory of Intracellular Membrane Dynamics, Graduate School of Frontier

Biosciences, Osaka University, Osaka, Japan, ${ }^{\mathrm{b}}$ Department of Genetics, Graduate School

of Medicine, Osaka University, Osaka, Japan

${ }^{1}$ Current address: Laboratory of Molecular Traffic, Institute for Molecular and Cellular

Regulation, Gunma University, Gunma, Japan

Correspondence to Tamotsu Yoshimori

Laboratory of Intracellular Membrane Dynamics, Graduate School of Frontier

Biosciences, Osaka University, 2-2 Yamadaoka, Suita, Osaka 565-0871, Japan

Tel: +81-6-6879-3580, Fax: +81-6-6879-3589, E-mail: tamyoshi@ fbs.osaka-u.ac.jp 


\section{Abstract}

Autophagy is a bulk degradation system that is induced under stress conditions such as nutrient deprivation. Selective autophagy, including xenophagy and mitophagy, is believed to play important roles in the development of several diseases. Consequently, selective autophagy represents a potential therapeutic target. Recent work showed that the lysosome, a membrane-bound acidic organelle, is selectively sequestered by autophagy when its membrane is injured; this phenomenon is called "lysophagy". Lysosomes can be injured by diverse causes, including amyloid proteins and mineral crystals such as silica and monosodium urate, which would trigger neurodegeneration and other diseases. In this section, we provide an overview of methods for monitoring lysophagy in mammalian cultured cells. These methods can be used to evaluate the involvement of molecules of interest in selective autophagy, and in screens aimed at identifying novel proteins engaged in selective autophagy. 


\section{Introduction}

Autophagy is an intracellular degradation system conserved from yeast to mammals. A cup-shaped double-membrane structure named the isolation membrane (also called the phagophore) forms in the cytoplasm in response to stress such as nutrient deprivation. The isolation membrane expands and sequesters cytosolic materials and organelles, and subsequently closes to form an autophagosome. After autophagosomes form, they fuse with the lysosome to degrade their contents [1-3]. Beyond the physiological roles of autophagy in production of energy to help cells survive starvation and stress, defects in autophagy cause a variety of diseases such as neurodegenerative disorders, diabetes, and heart failure $[4,5]$.

Over 30 autophagy-related (Atg) genes have been discovered to date, most of which were identified by genetic screens using yeast. Autophagosome formation can be divided into several steps as follows [1,6,7]. (1) Initiation of autophagy: the uncoordinated 51-like kinase 1 (ULK1) complex is recruited to the autophagosome formation site after inactivation of the mammalian target of rapamycin (mTOR), followed 
by activation of the class III phosphatidylinositol 3-kinase (PI3K), leading to the formation of phosphatidylinositol 3-phosphate (PI3P) and recruitment of PI3P-binding proteins. (2) Vesicle nucleation: the transmembrane protein Atg9L1 participates in the nucleation of the autophagosome membrane. (3) Autophagosome formation: the C-terminus of LC3 (mammalian homologue of Atg8 in yeast) is conjugated to phosphatidylethanolamine (PE) to anchor the protein in membrane; this conjugation is mediated by a ubiquitin-like system consisting of Atg7 (E1 enzyme), Atg3 (E2 enzyme), and the Atg12-Atg5-Atg16L1 complex (E3 enzyme). (4) Elongation of the isolation membrane and degradation, in which a sealed autophagosome fuses with a lysosome.

Basal and starvation-induced autophagy are non-selective processes. However, autophagy also can target certain substrates including damaged organelles, invading bacteria and aggregate-prone proteins (selective autophagy), which plays important role in pathophysiological aspects. In particular, autophagy maintains intracellular homeostasis by capturing damaged mitochondria (mitophagy), aggregating proteins (aggrephagy), and bacteria invading into cells (xenophagy), thereby removing materials "toxic" for cells $[8,9]$. In the last few years, we and other groups have proposed that the 
lysosome, an acidic membrane-bound organelle, can itself be targeted by autophagy if it is damaged $[10,11]$. We demonstrated that damaged lysosomes, which are harmful to cells, are sequestered by autophagosomes in a process we call "lysophagy". Each type of selective autophagy shares a common feature: some proteins on the damaged organelle membrane are ubiquitinated, Atg proteins such as Atg16L1 and LC3 are recruited directly (via ubiquitin binding domains) or indirectly (via adaptor proteins), and then the isolation membrane elongates to form autophagosomes that engulf the damaged organelle $[8,12]$. Therefore, ubiquitination seems to function as the initiation factor for selective autophagy.

Because lysophagy has been reported only recently by a few groups, including ours, we felt it would be useful to describe detailed methods for detecting lysophagy in cultured cells. We introduce such methods here. 


\section{Description of methods}

\subsection{Overview}

The lysosome is an acidic organelle and the major digestive compartment in all eukaryotic cells. Lysosomes contain membrane proteins such as lysosome-associated membrane protein 1 (LAMP1), as well as more than 60 resident soluble hydrolases including cathepsins, which are proteases and function effectively in the acidic environment of lysosomal lumen ( $\mathrm{pH} 4-5)$ [13,14]. The release of large amounts of cathepsins from lysosomes into the cytosol results in apoptotic cell death, either via direct activation of caspases or via digestion of a pro-apoptotic Bcl-2 family member Bid [13,15-19]. Lysosomal rupture also induces the release of protons and calcium from the lysosomal compartment, interfering with cellular functions $[15,20]$.

The remnants of damaged lysosomes are also harmful to cells. Cells have no way to maintain cellular homeostasis if damaged lysosomes that lack digestive ability are not removed, because the total number of intracellular lysosomes does not alter even if some of them become dysfunctional [10]. In addition, the remaining damaged lysosomes 
may aggressively compromise the health of cells, e.g., by continuous activation of inflammation response. Removal of the damaged lysosomes is achieved by selective autophagy [10]. Therefore, lysophagy plays an important role in preserving the number of functional lysosomes and maintaining cellular homeostasis. In fact, proximal tubule-specific autophagy-deficient mice exhibit severe nephropathy under hyperuricemia, which induces lysosomal damage in kidney [10]. Thus, lysophagy plays an essential role in suppressing the development of acute hyperuricemic nephropathy in mice.

Galectins, small soluble proteins that localize to cytoplasm and nucleus, are defined by their affinity for $\beta$-galactose-containing carbohydrates $[21,22]$. $\beta$-galactose-containing glycoconjugates are typically found on the cell surface and in the lumen of endocytic compartments such as endosomes, lysosomes, and the Golgi apparatus. Recent work showed that galectin-3 accumulates at endomembranes damaged by bacterial infection [23-25]. Moreover, we demonstrated that lysosomal rupture induces the recruitment of galectin-3 to injured lysosomes [10]. In addition to galectin-3, galectin- 8 and -9 are also recruited to damaged membranes by bacterial invasion [26]. In 
particular, galectin-8 binds to NDP52, an autophagy receptor with ubiquitin-binding activity. These findings suggest that galectin-3, -8 , and -9 are useful markers for damaged endomembranes.

A variety of materials, including silica [27], monosodium urate [28], bacterial toxins [29,30], lipids [31], $\beta$-amyloid [32], and lysosomotropic agents such as L-Leucyl-L-leucine methyl ester (LLOMe) [10] can break the lysosomal membrane. (although LLOMe is activated only in lysosomes so that only injures them, the other materials may damage other endomembranes, for example endosomes as well) . In vivo, these substances often cause diseases, including Parkinson's disease and hyperuricemic nephropathy $[28,33,34]$. In this section, we introduce methods for detection of lysophagy caused by LLOMe. LLOMe, a lysosomotropic reagent that is taken up via endocytosis [35], causes apoptosis of immune cells such as natural killer cells and macrophages. Internalized LLOMe is converted to (LeuLeu) $)_{n}$-OMe $(n>3)$ through dipeptide peptidase I, which functions as a thiol protease in lysosomes [36,37], and physiologically hydrolyzes glycyl-L-phenylalanine 2-naphthylamide [38]. Furthermore, LLOMe treatment robustly provokes the rupture of lysosomal membrane in a number of cell lines, 
including NIH3T3 and mouse embryonic fibroblasts (MEFs) [10], even though the effective concentration is higher $(1000 \mu \mathrm{M})$ than that in immune cells such as HL-60 $(100-250 \mu \mathrm{M})$. Silicon dioxide $\left(\mathrm{SiO}_{2}\right)$ can also induce the rupture of the lysosomal membrane in alveolar macrophages, resulting in activation of lung inflammation $[27,39]$.

\subsection{Cell culture, treatment and immunofluorescence}

\subsubsection{LLOMe treatment}

1. Culture MEF or NIH3T3 cells in Dulbecco's Modified Eagle Medium (DMEM) with $10 \%$ fetal bovine serum (FBS) and 100 units $/ \mathrm{ml}$ penicillin and $100 \mu \mathrm{g} / \mathrm{ml}$ streptomycin at $37^{\circ} \mathrm{C}$ in a $5 \% \mathrm{CO}_{2}$ incubator.

2. Coat glass coverslips (Matsunami glass, $12-\mathrm{mm}$ round) with $0.1 \mathrm{mg} / \mathrm{ml}$ collagen (Nitta gelatin, Cell matrix Type I-C) for at least $10 \mathrm{~min}$ at room temperature, and then wash

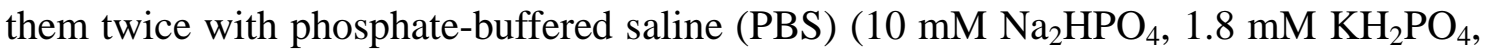
$137 \mathrm{mM} \mathrm{NaCl}, 2.7 \mathrm{mM} \mathrm{KCl}, \mathrm{pH}$ 7.4). 
3. Seed cells on sterilized coated coverslips in a 24-well plate in DMEM with $10 \%$ FBS, such that they will become $60-70 \%$ confluent at the time of the experiment. Incubate the cells at $37^{\circ} \mathrm{C}$ in a $5 \% \mathrm{CO}_{2}$ incubator for $18-24 \mathrm{~h}$.

4. Discard the medium. To induce lysophagy, incubate the cells with 100-1000 $\mu \mathrm{M}$ LLOMe, depending on the cell line, in DMEM with 10\% FBS for $1 \mathrm{~h}$. A suggested stock solution is $333 \mathrm{mM}$ LLOMe (Sigma, L7393) dissolved in ethanol. The solution should be firmly sealed and stored at $-20^{\circ} \mathrm{C}$. To create the stock, dissolve $1.13 \mathrm{~g}$ LLOMe in $10 \mathrm{ml}$ Ethanol, and agitate the solution to dissolve the crystals completely. Because frequent opening of the same tubes leads to inactivation of the LLOMe, it is preferable to divide the stock solution into small aliquots.

5. Wash the cells twice with DMEM with $10 \%$ FBS and culture them at $37^{\circ} \mathrm{C}$ for several hours to allow lysophagy of the injured lysosomes. Because some types of cells (e.g., MEFs) are easily detached after LLOMe treatment, the cells should be washed gently. 
6. Wash the cells with PBS, and then fix them in 4\% PFA in PBS for 15 min at room temperature. Wash the cells twice with PBS.

7. Permeabilize the cells with $50 \mu \mathrm{g} / \mathrm{ml}$ digitonin (Wako, 043-21371) in $0.1 \%$ gelatin (Wako, 043-21371) in PBS for 10 min. Wash them twice with PBS.

8. Block the cells with $0.1 \%$ gelatin in PBS for 30 min at room temperature.

9. Incubate the cells on coverslips with $30 \mu \mathrm{l}$ of rat monoclonal anti-galectin-3 antibody (Santa Cruz Biotechnology, sc-23938) diluted 1:1000 in 0.1\% gelatin in PBS for $1 \mathrm{~h}$ at room temperature. Wash the samples three times with PBS. For co-staining with LC3 (autophagosome marker), dilute rabbit polyclonal anti-LC3 antibody (MBL, PM036) at 1:1000 together with anti-galectin-3 antibody in $0.1 \%$ gelatin in PBS.

10. Incubate the cells on coverslips with $30 \mu 1$ of secondary antibody (e.g., Alexa Fluor 
488-conjugated anti-rat IgG antibody), diluted 1:5000, and DAPI (Invitrogen, D3571;

final concentration, $300 \mathrm{nM}$ ) in $0.1 \%$ gelatin in PBS for 40 min at room temperature.

Wash the samples three times with PBS.

11. Wash the coverslips with distilled water once and remove extra water. Mount the samples on glass slides with $5 \mu$ l Prolong Gold (Molecular Probes, P36930), a mounting reagent.

12. Observe galectin-3 staining by fluorescence microscopy. Count the number of galectin-3 dots per cell. Analyze the images using software such as CellProfiler (http://www.cellprofiler.org); we recommend this user-friendly free software because of its high performance.

\subsubsection{Silica treatment}

1. Follow steps $1-3$ in section 2.2.1. 
2. Discard the medium, and incubate the cells with $62.5-500 \mu \mathrm{g} / \mathrm{ml}$ silicon dioxide dissolved in DMEM with 10\% FBS for 1-3 h, depending on the cells used. A suggested stock solution is $1 \mathrm{mg} / \mathrm{ml}$ silicon dioxide (Sigma, S5631) dissolved in PBS and stored at $-20^{\circ} \mathrm{C}$. Because silicon dioxide crystals aggregate during storage, the stock solution should be resuspended before use by passage through a 25 -gauge needle.

3. Follow steps 5-12 in section 2.2.1.

\subsection{Observe GFP-Galectin-3}

Endogenous galectin-3 staining may exhibit a low signal-to-noise ratio, resulting in inaccurate dot counting. To overcome this obstacle, exogenous expression of GFP-galectin-3 is a useful alternative technique. A more precise dot count can be achieved by using cells stably expressing GFP-galectin-3. Using such cells, follow the protocol described above for immunostaining with anti-LC3 antibody. 


\section{4 tfGalectin-3 assay}

To observe sequential removal of damaged lysosomes, we developed a probe for the detection of damaged lysosomes, mRFP-GFP tandem-tagged Galectin-3 (tandem fluorescent-tagged Galectin-3, tfGal3) [10]. This probe is based on the tfLC3 assay that we developed previously [40]. GFP fluorescence is attenuated and degraded by lysosomal acidic $\mathrm{pH}$ and hydrolases, whereas mRFP fluorescence is comparatively stable even under acidic conditions. Thus, tfGal3 enables monitoring of the $\mathrm{pH}$ change in damaged lysosomes. As shown in Fig. 1, under normal conditions, tfGal3 localizes diffusely into the cytoplasm. tfGal3 localizes inside lysosomes after they are ruptured by (e.g.) LLOMe treatment, and most Gal3 puncta contain both red and green signals. After damaged lysosomes are sequestered by the autophagosomes, which subsequently fuse with intact lysosomes, tfGal3 displays only a red signal, because GFP fluorescence is quenched in an acidic environment. Use cells stably expressing tfGal3 and follow the protocol described above for immunostaining of LC3. 


\section{Results}

\subsection{Galectin-3 dot count}

We incubated MEFs with LLOMe for $3 \mathrm{~h}$, and then observed the recruitment of galectin-3

and LC3 to damaged lysosomes. Upon LLOMe treatment, galectin-3 accumulated in LAMP1-positive dots (Fig. 2). LC3 was also recruited to the damaged lysosomes (Galectin-3-positive dots). These results show that autophagosome formation occurs when lysosomes are damaged by LLOMe treatment.

Next, we investigated whether the ruptured lysosomes were restored in an autophagy-dependent manner. Cells were treated with $1000 \mu \mathrm{M}$ LLOMe for $1 \mathrm{~h}$, and after washout, the cells were cultured for further $24 \mathrm{~h}$ in the absence of LLOMe. We quantified the GFP-galectin-3-positive LAMP1 puncta at the indicated time points. Damaged lysosomes (Galectin-3-positive dots) were significantly less abundant $10 \mathrm{~h}$ after LLOMe washout, and completely disappeared in $24 \mathrm{~h}$ (Fig. 3). However, in cells expressing an Atg4B mutant (Atg4B ${ }^{\mathrm{C} 74 \mathrm{~B}}$ ), which hampers the lipidation of LC3 paralogues and strongly suppresses autophagy [41], the reduction of GFP-galectin-3-positive puncta was 
inhibited. These data suggest that the recovery of damaged lysosomes depends on autophagy.

\section{2 tfGal3 assay}

As described above, tfGal3 is a useful tool for monitoring $\mathrm{pH}$ changes when lysosomes are injured. As shown in Fig. 4, both green and red signals were observed in control cells, as well as in cells expressing the Atg4B mutant, immediately after LLOMe treatment (0 h). However, $24 \mathrm{~h}$ after LLOMe washout, GFP signals had disappeared in control cells, whereas most GFP puncta persisted in Atg4B mutant cells. This result indicates that the GFP signal of tfGal3 was attenuated in an autophagy-dependent manner. 


\section{Conclusions}

In this section, we described a basic protocol for monitoring lysophagy by using fluorescent proteins-tagged Galectin. Defect in lysophagy causes severe nephropathy under hyperuricemia in mice [10] and both the increase of cell death and the decrease of cell division in cultured cells (unpublished data). Thereby, functional assays (for example cell survival) would be useful to assess the importance of autophagy in response to endomembrane damage in addition to the Galectin assay.

When a compound is used to induce lysophagy (similar to the induction of mitophagy), most cells exposed to the compound exhibit lysosomal rupture. Therefore, we can carry out biochemical analyses using whole cell lysates, increasing the likelihood that we can find novel molecules and signal cascades required for selective autophagy. Because the NIH3T3 and MEF cells used in this protocol are typical mammalian cells, we predict that the methods described here could be successfully applied to other types of mammalian cells. Because the lysophagy field is still developing, it remains unclear whether the molecular mechanism underlying this process is similar to that of other types 
of selective autophagy, such as xenophagy. Further studies will be required in order to clarify the detailed mechanism of lysophagy. 


\section{Acknowledgements}

This research was supported in part by the Ministry of Education, Culture, Sports,

Science and Technology (MEXT) of Japan, by Japan Science and Technology Agency

CREST. 


\section{Figure Legends}

Fig. 1.tfGal3 assay system. (A) Schematic representation of tandem fluorescence-tagged Galectin-3 (tfGal3). (B) Schematic diagram of tfGal3 assay. tfGal3 localizes to the cytoplasm under normal conditions. Once the lysosomal membrane is injured, tfGal3 accumulates inside the ruptured lysosomes (green- and red-positive dots). When the lysosomal membrane is sequestered by autophagosome, which subsequently fuses with intact lysosomes, the lysosome lumen becomes acidic and only the red signal of tfGal3 remains.

Fig. 2. The recruitment of LC3 to galectin-3-positive damaged lysosomes. $\operatorname{Atg} 7^{+/+}$and Atg $7^{-/-}$MEF cells stably expressing GFP-galectin-3 were treated with $1000 \mu \mathrm{M}$ LLOMe for $3 \mathrm{~h} . \operatorname{Atg} 7^{+/+}$cells, but not $A \operatorname{tg} 7^{-/-}$cells, exhibited strong accumulation of LC3 at damaged lysosomes (GFP-galectin-3-positive dots). Bars; $10 \mu \mathrm{m}$. [Adapted from Maejima et al. [10]. Copyright 2013 Maejima et al. The EMBO Journal. 32: 2336-2347. doi: 10.1038/emboj.2013.171.] 
Fig. 3. Reduction of galectin-3 puncta is dependent on autophagy. (A) NIH3T3 cells stably expressing GFP-galectin-3 and either empty vector (control) or mStrawberry-Atg4B ${ }^{\mathrm{C} 74 \mathrm{~A}}$ (Atg4B mutant) were treated with $1000 \mu \mathrm{M}$ LLOMe for $1 \mathrm{~h}$. The cells were fixed at the indicated time points after LLOMe washout, and then stained with anti-LAMP1 antibodies and DAPI (Blue). (B) Quantitation of the data shown in A. Cells expressing the Atg4B mutant exhibited reduced recovery of damaged lysosomes relative to control cells. Bars; $20 \mu \mathrm{m}$. [Adapted from Maejima et al. [10]. Copyright 2013 Maejima et al. The EMBO Journal. 32: 2336-2347. doi: 10.1038/emboj.2013.171.]

Fig. 4. Lysophagy assay using tfGal3. HeLa cells transfected with tfGal3 and either empty vector (control) or FLAG-tagged $A \operatorname{tg} 4 \mathrm{~B}^{\mathrm{C} 74 \mathrm{~A}}$ (Atg4B mutant) were treated with $1000 \mu \mathrm{M}$ LLOMe for $1 \mathrm{~h}$. The cells were fixed at the indicated time points after LLOMe washout, and then observed by confocal microscopy. Bars; $20 \mu \mathrm{m}$. [Adapted from Maejima et al. [10]. Copyright 2013 Maejima et al. The EMBO Journal. 32: 2336-2347. doi: 10.1038/emboj.2013.171.] 


\section{References}

[1] Mizushima N, Yoshimori T, Ohsumi Y. Annu Rev Cell Dev Biol. 27 (2011) 107-132.

[2] Mizushima N, Komatsu M. Cell. 147 (2011) 728-741.

[3] Lamb CA, Yoshimori T, Tooze SA. Nat Rev Mol Cell Biol. 14 (2013) 759-774.

[4] Mizushima N, Levine B. Nat Cell Biol. 12 (2010) 823-830.

[5] Schneider JL, Cuervo AM. Curr Opin Genet Dev. 26C (2014) 16-23.

[6] Mackeh R, Perdiz D, Lorin S, Codogno P, Poüs C. J Cell Sci. 126 (2013) 1071-1080.

[7] Suzuki K, Ohsumi Y. FEBS Lett. 584 (2010) 1280-1286.

[8] Stolz A, Ernst A, Dikic I. Nat Cell Biol. 16 (2014) 495-501.

[9] Randow F, Youle RJ. Cell Host Microbe. 15 (2014) 403-411.

[10] Maejima I, Takahashi A, Omori H, Kimura T, Takabatake Y, Saitoh T, et al. Embo J. 32 (2013) 2336-2347.

[11] Hung Y-H, Chen LM-W, Yang J-Y, Yang WY. Nat Commun. 4 (2013) 2111.

[12] Fujita N, Morita E, Itoh T, Tanaka A, Nakaoka M, Osada Y, et al. J Cell Biol. 203 (2013) $115-128$.

[13] Appelqvist H, Wäster P, Kågedal K, Öllinger K. J Mol Cell Biol. 5 (2013) 214-226.

[14] Saftig P, Klumperman J. Nat Rev Mol Cell Biol. 10 (2009) 623-635.

[15] Boya P, Kroemer G. Oncogene. 27 (2008) 6434-6451.

[16] Ishisaka R, Utsumi T, Yabuki M, Kanno T, Furuno T, Inoue M, et al. FEBS Lett. 435 (1998) 233-236.

[17] Conus S, Perozzo R, Reinheckel T, Peters C, Scapozza L, Yousefi S, et al. J Exp Med. 205 (2008) 685-698.

[18] Cirman T, Oresić K, Mazovec GD, Turk V, Reed JC, Myers RM, et al. J Biol Chem. 279 (2004) 3578-3587.

[19] Appelqvist H, Johansson A-C, Linderoth E, Johansson U, Antonsson B, Steinfeld R, et al. Ann Clin Lab Sci. 42 (2012) 231-242.

[20] Mirnikjoo B, Balasubramanian K, Schroit AJ. J Biol Chem. 284 (2009) 6918-6923.

[21] Houzelstein D, Gonçalves IR, Fadden AJ, Sidhu SS, Cooper DNW, Drickamer K, et al. Mol Biol Evol. 21 (2004) 1177-1187.

[22] Leffler H, Carlsson S, Hedlund M, Qian Y, Poirier F. Glycoconj J. 19 (2004) 433-440. 
[23] Dupont N, Lacas-Gervais S, Bertout J, Paz I, Freche B, Van Nhieu GT, et al. Cell Host Microbe. 6 (2009) 137-149.

[24] Paz I, Sachse M, Dupont N, Mounier J, Cederfur C, Enninga J, et al. Cellular Microbiology. 12 (2010) 530-544.

[25] Ray K, Bobard A, Danckaert A, Paz-Haftel I, Clair C, Ehsani S, et al. Cellular Microbiology. 12 (2010) 545-556.

[26] Thurston TLM, Wandel MP, Muhlinen von N, Foeglein Á, Randow F. Nature. 482 (2012) 414-418.

[27] Hornung V, Bauernfeind F, Halle A, Samstad EO, Kono H, Rock KL, et al. Nat Immunol. 9 (2008) 847-856.

[28] Emmerson BT, Cross M, Osborne JM, Axelsen RA. Kidney Int. 37 (1990) 36-43.

[29] Molinari M, Galli C, Norais N, Telford JL, Rappuoli R, Luzio JP, et al. J Biol Chem. 272 (1997) 25339-25344.

[30] Prince LR, Bianchi SM, Vaughan KM, Bewley MA, Marriott HM, Walmsley SR, et al. J Immunol. 180 (2008) 3502-3511.

[31] Duewell P, Kono H, Rayner KJ, Sirois CM, Vladimer G, Bauernfeind FG, et al. Nature. 464 (2010) 1357-1361.

[32] Ditaranto K, Tekirian TL, Yang AJ. Neurobiol Dis. 8 (2001) 19-31.

[33] Waisman J, Mwasi LM, Bluestone R, Klinenberg JR. Am J Pathol. 81 (1975) 367-378.

[34] Dehay B, Bové J, Rodríguez-Muela N, Perier C, Recasens A, Boya P, et al. J Neurosci. 30 (2010) 12535-12544.

[35] Thiele DL, Lipsky PE. J Exp Med. 172 (1990) 183-194.

[36] Thiele DL, Lipsky PE. J Immunol. 148 (1992) 3950-3957.

[37] Uchimoto T, Nohara H, Kamehara R, Iwamura M, Watanabe N, Kobayashi Y. Apoptosis. 4 (1999) 357-362.

[38] Jadot M, Colmant C, Wattiaux-De Coninck S, Wattiaux R. Biochem J. 219 (1984) 965-970.

[39] Mossman BT, Churg A. Am J Respir Crit Care Med. 157 (1998) 1666-1680.

[40] Kimura S, Noda T, Yoshimori T. Autophagy. 3 (2007) 452-460.

[41] Fujita N, Hayashi-Nishino M, Fukumoto H, Omori H, Yamamoto A, Noda T, et al. Mol Biol Cell. 19 (2008) 4651-4659. 
A

\section{mRFP - EGFP - Galectin-3}

B

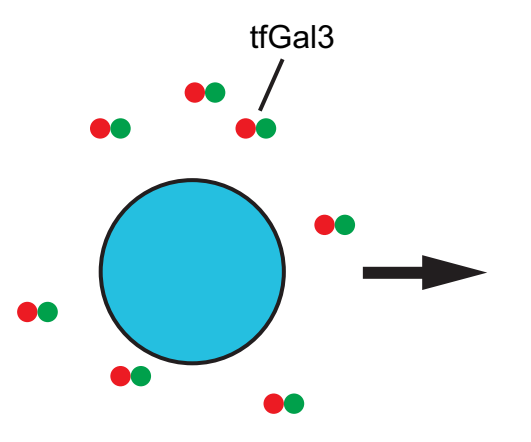
Lysosome
( $\mathrm{pH}$ : acidic)
Ruptured-lysosome
(pH: neutral)

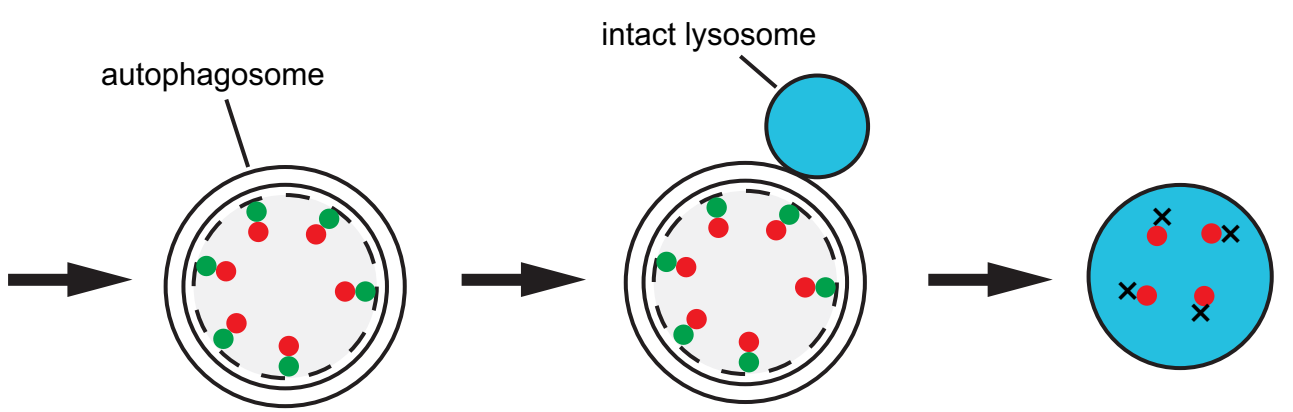

Sequestered lysosome
Sequestered lysosome fusue with intact lysosome

Lysosome ( $\mathrm{pH}$ : acidic)

Fig. 1 
Non-treated

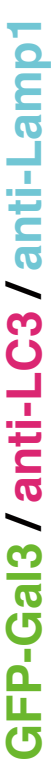
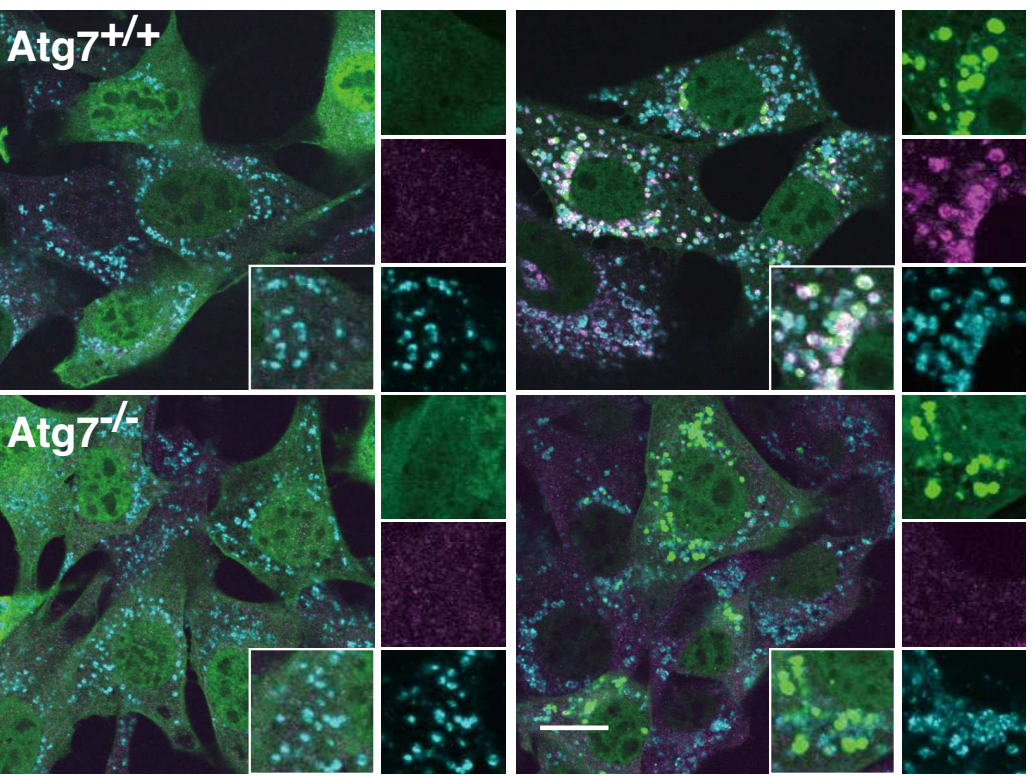

Fig. 2 
A Time after LLOMe washout $(\mathrm{h})$
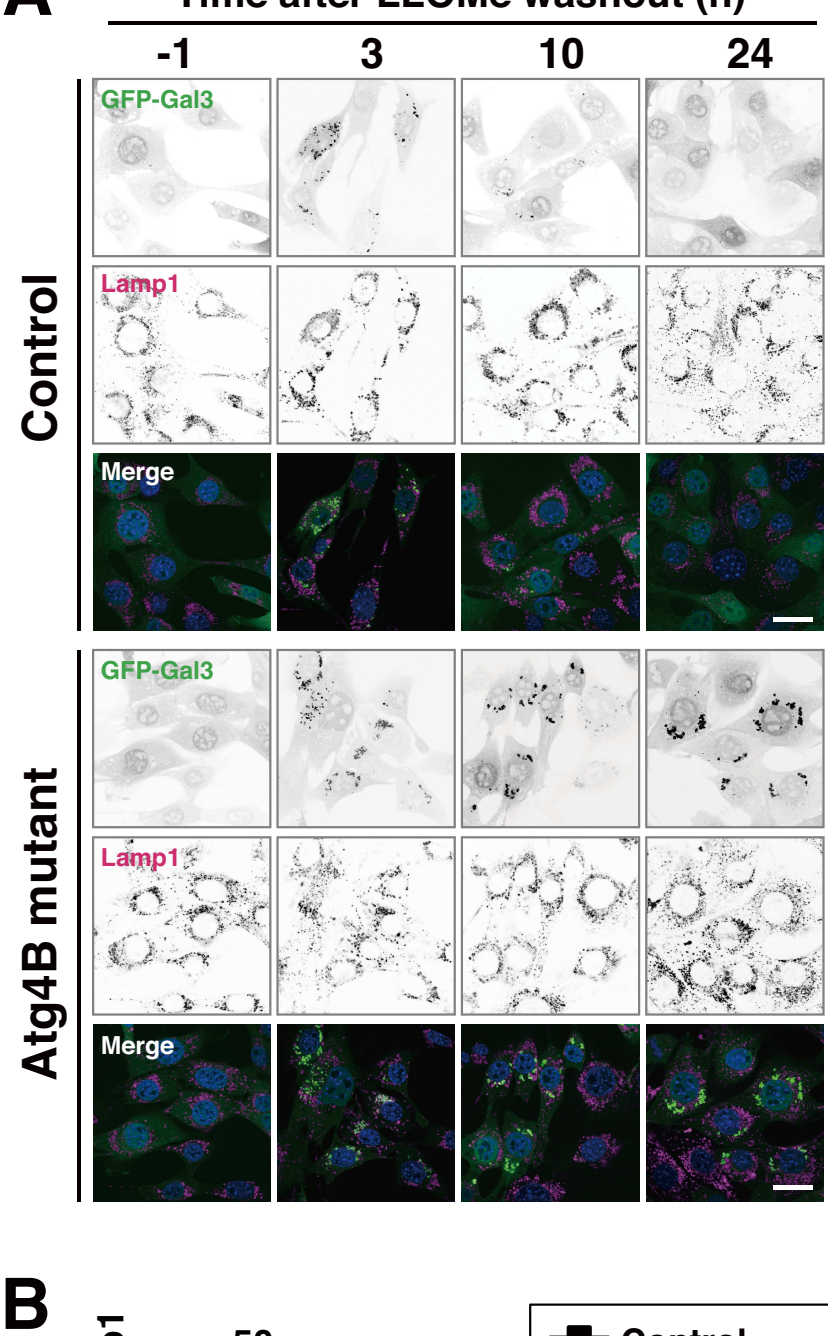

High magnification
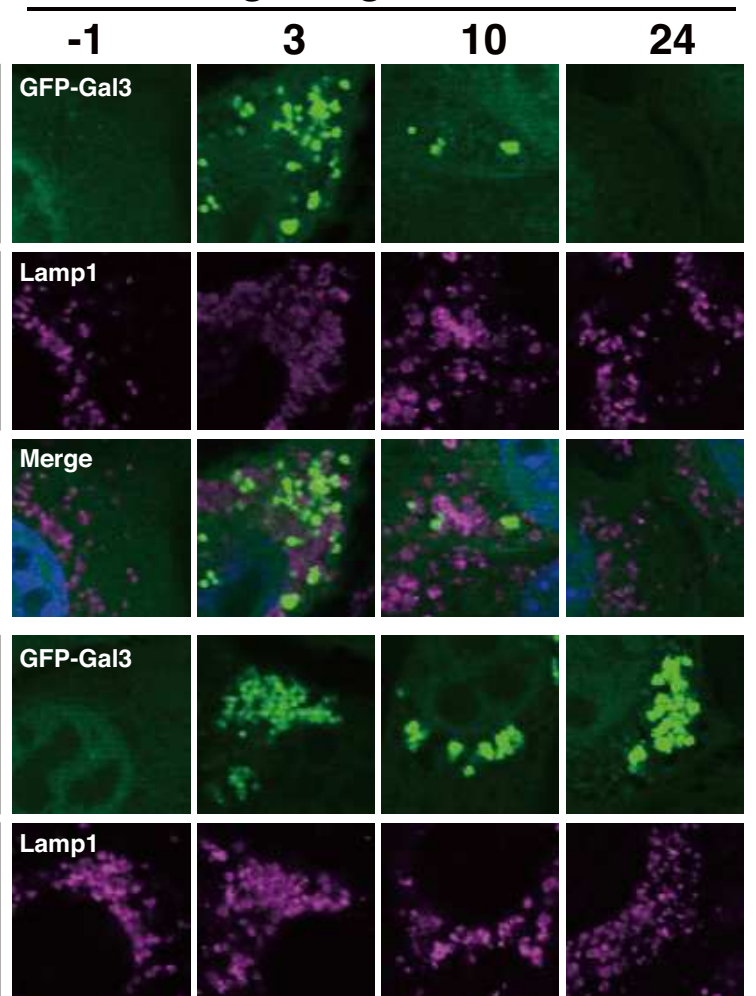

Merge
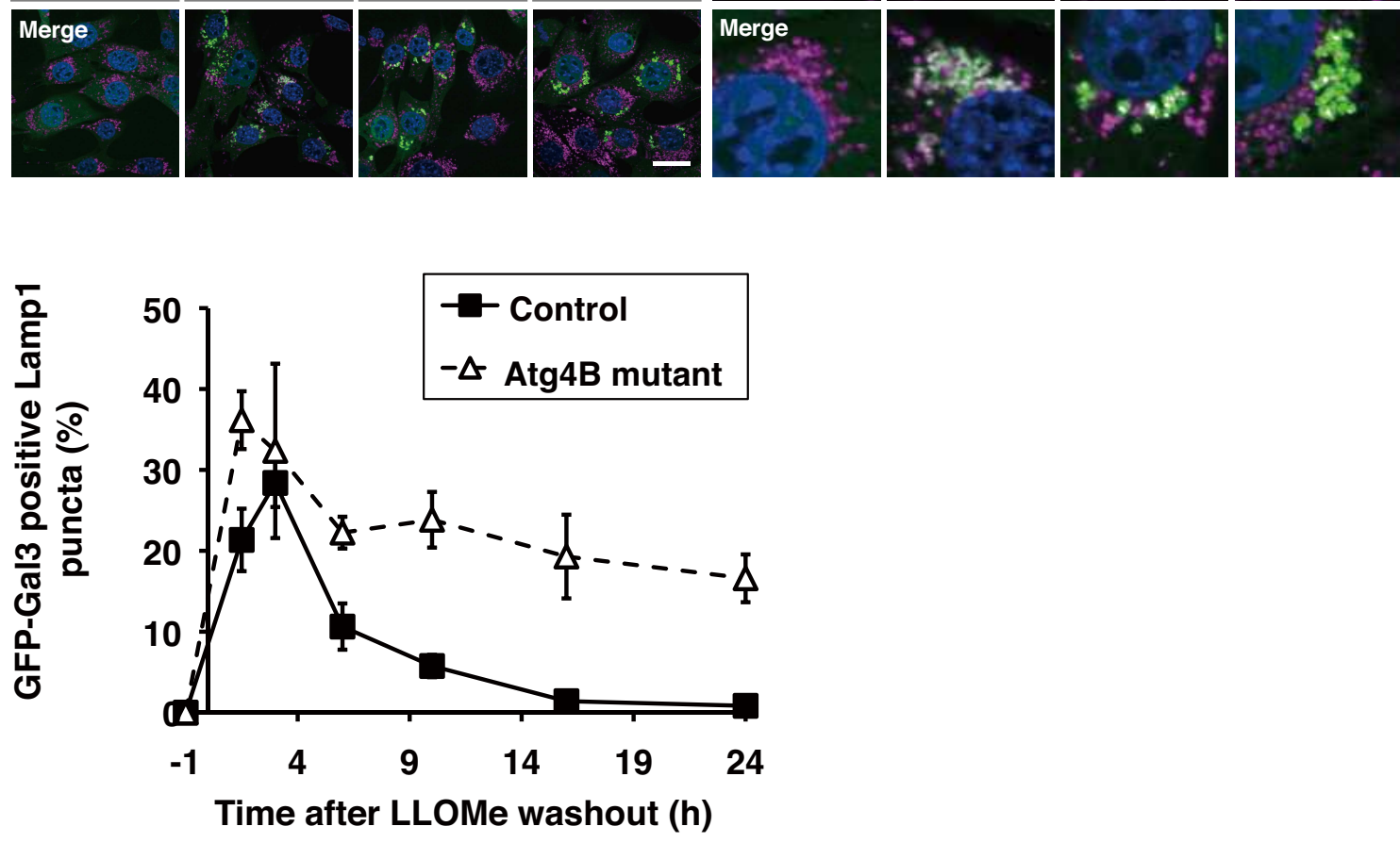

Fig. 3 
